\title{
Discussion on Anisotropic Magnetic Shielding Effectiveness of Shielded Package Analyzed by Magnetic Circuit Calculation
}

\author{
K. Yamada, Y. Fuji, and M. Hara \\ Corporate Research \& Development Center, Research \& Development Div., Toshiba Corp., \\ 1 Komukai-toshiba-cho, Saiwai-ku, Kawasaki 212-8582, Japan
}

\begin{abstract}
This paper presents millimeter-scale small magnetic shields for electrical components such as magnetic sensors or magnetic random-access memories (MRAM). A prototype of a shielded package consisting of two shield pieces is fabricated by using a MEMS package assembly line. The shielded package exhibits anisotropic magnetic shielding effectiveness (MSE). Two magnetic circuit models are introduced to explain the cause of the anisotropic MSE. The models reveal that magnetic flux flow and magnetic reluctances are changed by an external magnetic field, which causes anisotropic MSE.
\end{abstract}

Key words: Magnetic field measurement, sensor, component-level shield, shielded package, magnetic circuit

\section{Introduction}

The emergence of the Internet of Things is spurring the need for small, high-sensitivity, low-power sensors. Magnetic elements having an inverse magnetostrictive effect are expected to be applied for pressure sensors and vibration sensors offering high sensitivity and low power consumption ${ }^{1-2}$. Magnetic random-access memory (MRAM) realizes nonvolatile main memory with low power consumption ${ }^{3)}$. However, these magnetic devices are prone to malfunction in the presence of external noise. Therefore, they must be covered by magnetic shields to reduce the noise level around the magnetic elements.

Component-level shields (CLSs) and board-level shields (BLSs) are applicable to magnetic devices, but BLSs need more space to cover many components and thus become large. In comparison, CLSs cover only magnetic devices and are small and cost-effective. In addition, CLSs can benefit board designers by relieving them of the burden of complicated shield designs.

Magnetic CLSs have been studied by some groups. Wang et al. analyzed cylindrical shells that offer a magnetic shielding effectiveness (MSE) of more than 60 $\mathrm{dB}^{4}$. Watanabe et al. analyzed a U-shaped magnetic CLS for the case that a one-directional external magnetic field is applied the shield ${ }^{5}$.

We prototyped a magnetic shielded package consisting of two pieces of magnetic shield as reported in Ref. 6). Because the package is exposed to external magnetic noise arriving from various directions, the directional dependence of the package's MSE is important. Therefore, we measured and analyzed the anisotropic MSE of the prototype. The size of the package was $9 \times 9 \times 3.5 \mathrm{~mm}^{3}$, which is too large for use as an electrical component. Furthermore, the package exhibited a high MSE with respect to the package's longitudinal direction but a low MSE with respect to its short direction. However, we did not understand why the package possessed anisotropic MSE.

This paper discusses the cause of the anisotropic MSE. We measure and analyze a new small shielded package prototype fabricated on a MEMS package assembly line. The volume of the package is $82 \%$ smaller than the prototyped package of Ref. 6).

Then, we introduce two magnetic circuit models to consider the mechanism of anisotropic MSE. The MSE was calculated with a magnetic circuit using a rotationally symmetric cylindrical shell shield that is described in Ref. 7). To apply a magnetic shield to electronic components, the shield must consist of two pieces of shield, and the shape of the shield must be rectangular from the viewpoint of assembly. However, to our knowledge, there are no reports of such a small rectangular magnetic shield being studied with magnetic circuit models. The introduced magnetic circuit models reveal that the magnetic reluctance of the gap between two pieces of shield and the magnetic reluctance in the package are the causes of anisotropic MSE.

\section{Fabricating prototype}

A cross-sectional image of the prototype magnetic shielded package is shown in Fig. 1. A three-directional geomagnetic sensor (HSCDTD008A, Alps Electric Co., Ltd.) is covered by an upper shield and a lower shield. The sensor is bonded on the lower shield. The upper shield is adhered on the lower shield by conductive adhesive. The thickness of the conductive adhesive is about 0.03 millimeters. The upper and lower shields are made of PC permalloy whose relative permeability is more than 60000 . The upper shield is made by bending a $0.1 \mathrm{~mm}$-thick PC permalloy plate. Because the shape of the upper shield is simpler than that of the shield in ref. 6 ), the package size can be small. The lower shield is made of a PC permalloy plate whose thickness is $0.2 \mathrm{~mm}$.

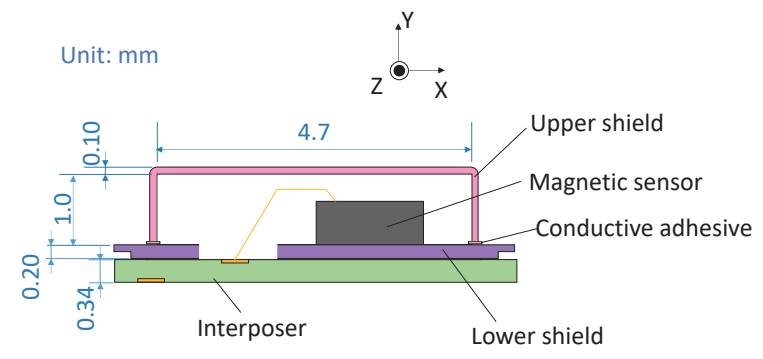

Fig. 1 Cross-sectional view of prototype of magnetic shielded package 
An interposer is also adhered to the lower shield. The base of the interposer is Flame Retardant Type 4 (FR4). The geomagnetic sensor is connected electrically to the electrodes of the interposer by wires.

Figs. 2 (a) and (b) show a top view and side view of the prototype package, respectively. The dimensions of the package are $5.0 \times 6.0 \times 1.7 \mathrm{~mm}^{3}$. The volume of the package is $82 \%$ smaller than the prototyped package of reference 6 ). The space covered by the upper and lower shields is $3.7 \times 4.7 \times 1.0 \mathrm{~mm}^{3}$.

Fig. 3 shows the process of assembling the package. First, the interposer is arranged as shown in Fig. 3 (a). The packages are assembled in a batch process so that the same conductive film patterns are lined up on the interposer. Then, the interposer and the lower shield are adhered with a heat press as shown in Fig. 3 (b). A hole in the lower shield for wires is arranged in advance. After that, the lower shield is diced as shown in Fig. 3 (c). The geomagnetic sensor is mounted on the lower shield, and wires are bonded as shown in Fig. 3 (d). Then, after dispensing the conductive adhesive on the lower shield, the upper shield is mounted on the conductive paste as shown in Fig. 3 (e). Last, the interposer is diced in a singulation process as shown in Fig. 3 (f). The difference from the conventional assembly process for MEMS microphones is the addition of Figs. 3 (b) and (c). Fig. 4 shows a photo of the prototype after assembly.

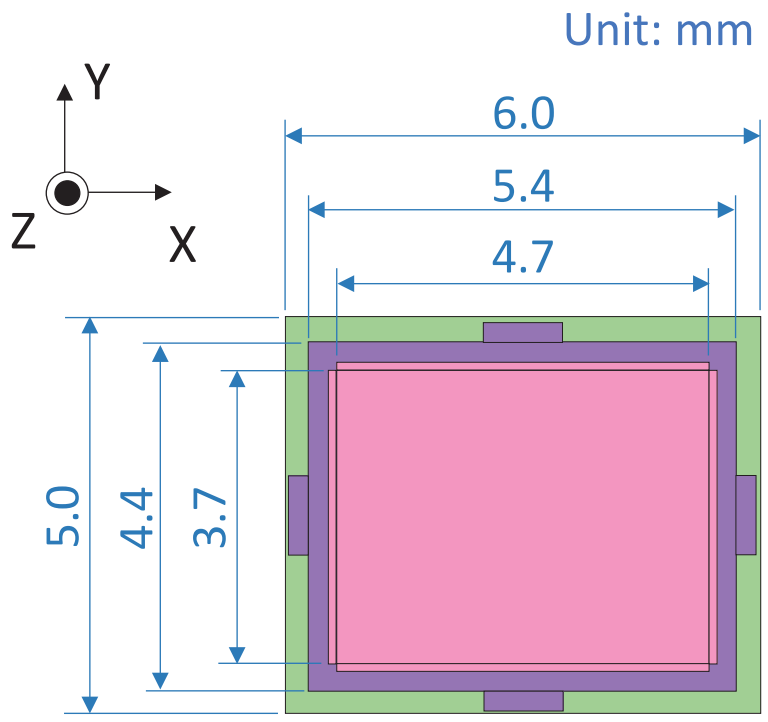

(a) Top view

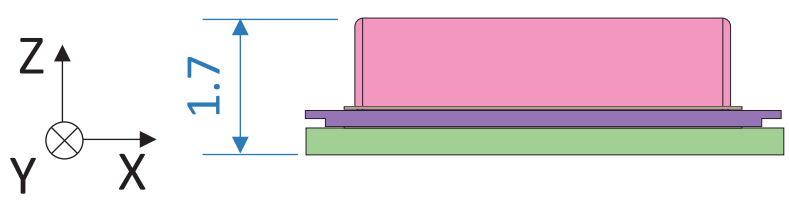

(b) Side view

Fig. 2 Dimensions of prototype magnetic shielded package

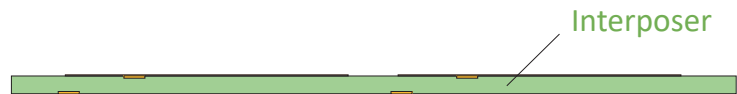

(a) Arranging the interposer

Lower shield

(b) Adhersion the interposer and lower shield

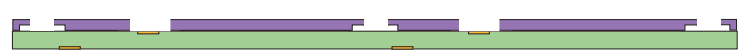

(c) Cutting the lower shield

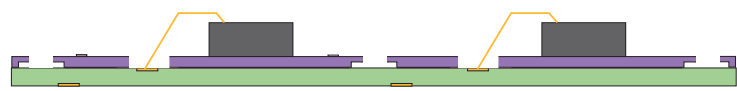

(d) Mounting the geomagnetic sensor and bonding wires

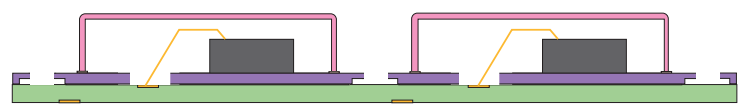

(e) Mounting upper shields

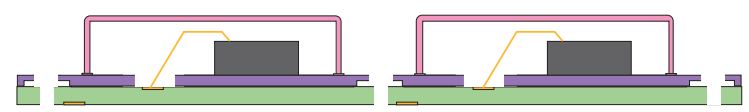

(f) Singulation

Fig. 3 Assembly flow of prototype magnetic shielded package

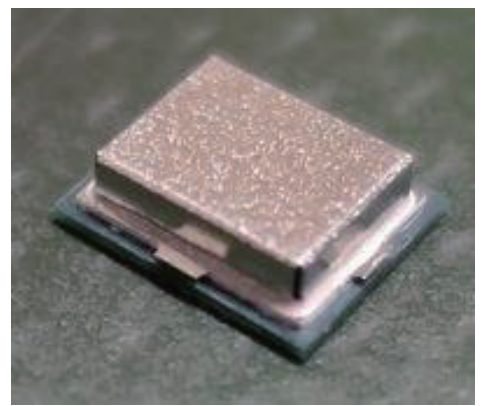

Fig. 4 Photo of prototype magnetic shielded package

\section{Analyzing and measuring anisotropic $M S E$}

\subsection{FEM simulation}

In this paper, similar to Ref. 6), MSE and the anisotropic MSE are defined as Equations (1) and (2), respectively.

$$
\operatorname{MSE}(\mathrm{dB})=-20 \log _{10} \frac{H_{w_{\_} \text {shield }}}{H_{\text {w/o_shield }}}
$$

$$
\left(\begin{array}{l}
H_{i n_{-} x} \\
H_{i n_{-} y} \\
H_{\text {in }_{-}}
\end{array}\right)=\left(\begin{array}{lll}
10^{-\frac{M S E x x}{20}} & 10^{-\frac{M S E x y}{20}} & 10^{-\frac{M S E x z}{20}} \\
10^{-\frac{M S E y x}{20}} & 10^{-\frac{M S E y y}{20}} & 10^{-\frac{M S E y z}{20}} \\
10^{-\frac{M S E z x}{20}} & 10^{-\frac{M S E z y}{20}} & 10^{-\frac{M S E z z}{20}}
\end{array}\right)\left(\begin{array}{l}
H_{e x_{-} x} \\
H_{e x_{-} y} \\
H_{e x_{-} z}
\end{array}\right)
$$


Here, $H_{W_{-} \text {shield }}$ is the magnetic field ( $H$-field) intensity detected by the geomagnetic sensor of the shielded package when the sensor is covered with the shields, and $H_{W / o}$ shield is the $H$-field intensity detected by the geomagnetic sensor when the sensor is not covered with the shields. In this paper, $H_{W /{ }_{0} \text { shield }}$ is equal to the external field.

Magnetic shields with the same dimensions as the prototype package were analyzed with a finite element method (FEM) magnetic field simulator. The FEM model is shown in Fig. 5. The model consisted of upper and lower shields. The dimensions of the shields were the same as those of the prototype. The gap between the shields was set to $0.03 \mathrm{~mm}$. A hole for wires was arranged in the lower shield. In this analysis, a DC external field was applied to the shields. This model included no nonmagnetic material that would influence flux. The intensity of $H$ ex was $1 \mathrm{~A} / \mathrm{m}$. $H_{\text {in }}$ was detected at the center of the geomagnetic sensor.

In this simulation, orthogonal three-directional $H_{\mathrm{ex}}$ was applied to the package. Fig. 6 shows the FEM analysis results. Fig. 6 also shows the measurement results described in the next section. $M S E_{x x}, M S E_{y x}$ and $M S E_{y y}$ were high, being more than $60 \mathrm{~dB}$. $M S E_{x y}, M S E_{x z}$, $M S E_{y z}, M S E_{z}$, and $M S E_{z y}$ were in the range of 50 to 60 dB. Only $M S E_{z z}$ was low, being $12 \mathrm{~dB}$.

Fig. 7 shows cut plane images of flux density. The cut plane was parallel to the XY plane and through the center of the sensor. Fig. 7 (a) shows the vector of flux density when an external field parallel to the $\mathrm{x}$-direction was applied. The magnetic sensor was arranged as indicated by the red dashed line. While magnetic flux flowed in the package around the hole for wires, little magnetic flux flowed around the sensor.

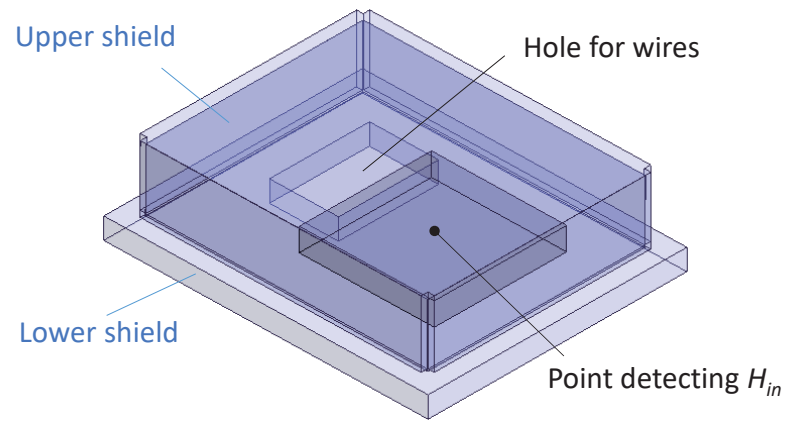

(a) Perspective view

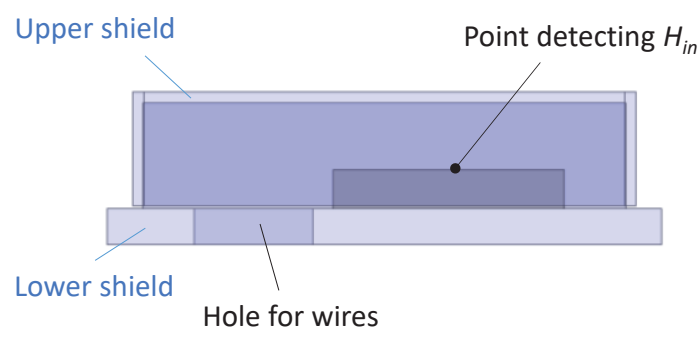

(b) Side view

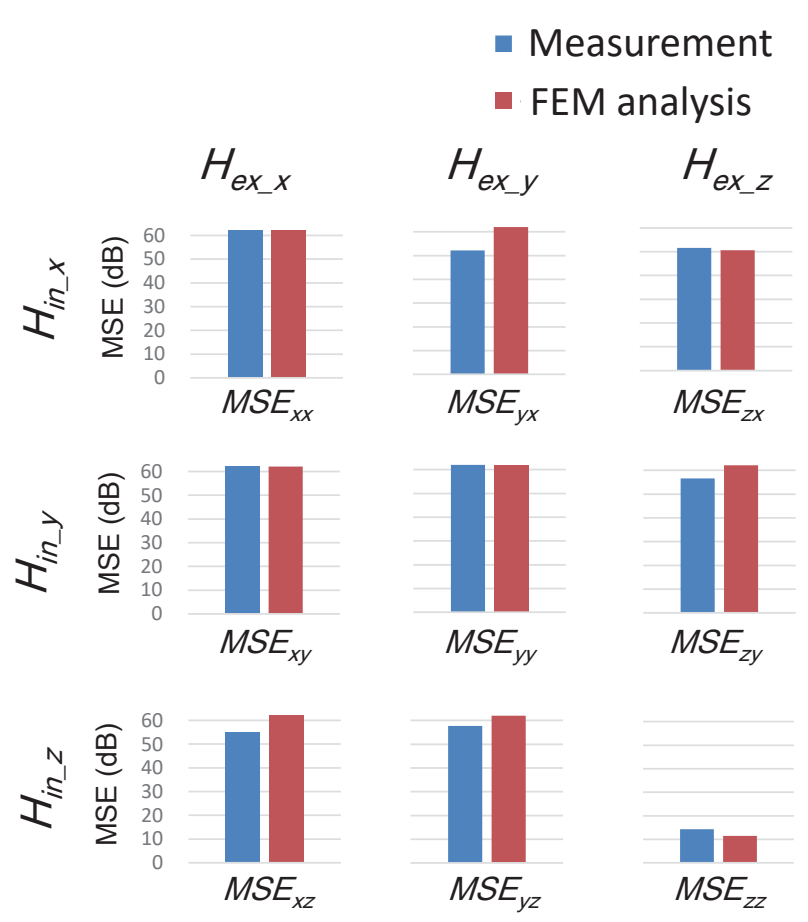

Fig. 6 Measurement and FEM analysis results of anisotropic MSE

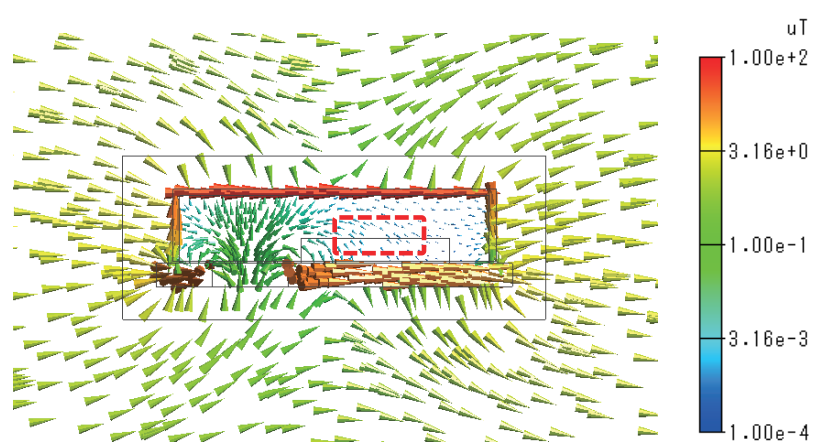

(a) $\mathrm{x}$-directional external magnetic field was applied

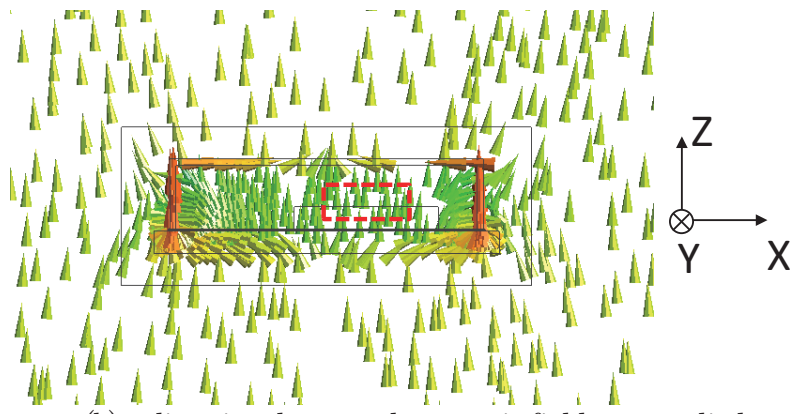

(b) z-directional external magnetic field was applied

Fig. 7 Cross-sectional view of magnetic flux density

On the other hand, when a z-directional external field was applied, comparatively high magnetic flux entered the package as shown in Fig. 7 (b). The flux was mainly along the $\mathrm{z}$-direction. From this simulation, the flux density largely depended on the direction of the external field.

Fig. 5 FEM simulation model 


\subsection{Measuring anisotropic $M S E$}

The anisotropic MSE of the prototype package was measured by using the same method in Ref. 6). An external field was applied by a Helmholtz coil, and the direction of the field was changed by rearranging the fixtures of the package. The internal field was sensed by the 3 -axis geomagnetic field sensor.

Fig. 6 shows the results of measuring anisotropic MSE. $M S E_{x x}, M S E_{y x}$, and $M S E_{y y}$ were more than $60 \mathrm{~dB}$, which is the same as the FEM analysis results. $M S E_{x y}, M S E_{x z}$, $M S E_{y z}, M S E_{z x}$, and $M S E_{z y}$ were more than $50 \mathrm{~dB}$. The differences between the measurement and analysis results for $M S E_{x y}, M S E_{x z}, M S E_{y z}, M S E_{z x}$, and $M S E_{z y}$ were less than $8 \mathrm{~dB}$. Because the magnetic field inside the package was very small, the difference was not a major issue.

$M S E_{z z}$ was the lowest, being $14 \mathrm{~dB} . M S E_{z z}$ for the comparatively large shielded package in Ref. 6) was 25 $\mathrm{dB}$. Though a detailed explanation will be provided in the next chapter, the low profile of the upper shield of this paper's prototype lead to a low $M S E_{z z}$.

\section{Mechanism of anisotropic MSE of shielded package}

Ref. 6) and Section 3 showed that the shielded packages possess anisotropic MSE. An understanding of why the packages have anisotropic MSE would be helpful for controlling MSE when shielded packages are designed. In this section, magnetic circuit models are introduced to facilitate discussion on the appearance of anisotropic MSE. Magnetic circuit calculation cannot consider leakage flux in detail and is inferior to FEM simulation in terms of accuracy. However, a magnetic circuit is useful for understanding the approximate values of magnetic reluctance and magnetic flux flow.

Fig. 8 shows a magnetic circuit model when $\mathrm{x}^{-}$ directional external field was applied. A magnetic flux source corresponding to an external magnetic noise source was set to $\phi_{\mathrm{x}} . R_{a x 1}$ and $R_{a x 2}$ were the magnetic

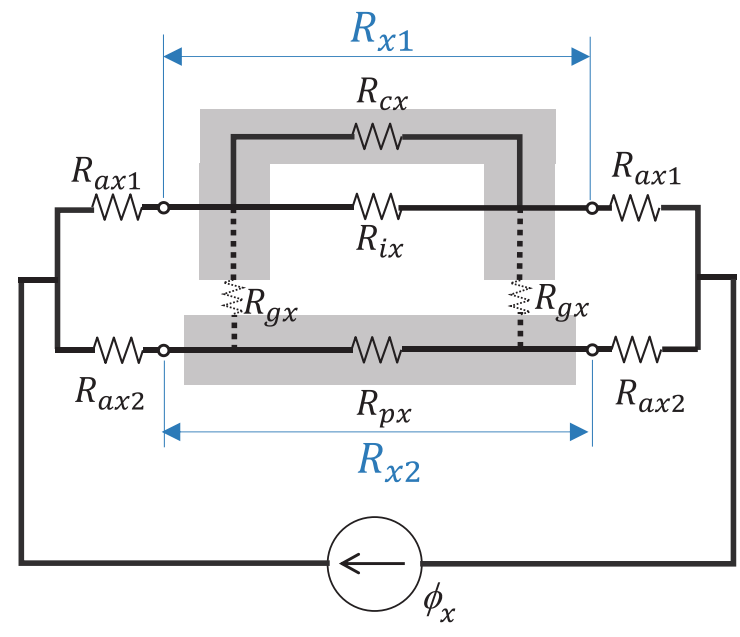

Fig. 8 Magnetic circuit model when $\mathrm{x}$-directional external field was applied reluctances of air outside the package. $R_{a x 1}$ and $R_{a x 2}$ were high. The ratio of magnetic flux passing through the upper shield and lower shield, which is the same value as the ratio of $R_{a x 2} / R_{a x 1}$, is difficult to estimate quantitatively. $R_{a x 1}$ and $R_{a x 2}$ are not discussed because they were magnetic reluctances outside the package. $R_{c x}$ was the magnetic reluctance of the upper shield, and $R_{i x}$ was the magnetic reluctance of magnetic flux from the upper shield through the air inside the package. $R_{p_{x}}$ was the magnetic reluctance of the lower shield. The magnetic reluctance of the gap between the upper and lower shields was expressed as $R_{g x}$. However, $R_{g_{x}}$ is not considered in the magnetic circuit calculation described below because magnetic flux does not pass through the gap. It is approximated that the magnetic flux into the package does not flow from the lower shield but from the upper shield.

The whole magnetic reluctance of the magnetic flux passing through the upper shield was set to $R_{x 1}$, and that passing through the lower shield was set to $R_{x 2}$. The length of the upper shield along the $\mathrm{x}$-direction was set to $a$, that along the $\mathrm{y}^{-}$direction was set to $b$, the height of the upper shield was $h$, and the permeability of the vacuum was set to $\mu_{o}$. Each resistance in Fig. 8 can be expressed as follows.

$$
\begin{aligned}
& R_{\mathrm{cx}}=(a+h) /\left(\mu_{0} \mu_{\mathrm{r}} b t_{\mathrm{c}}\right) \\
& R_{\mathrm{ix}}=a /\left(\mu_{0} b h\right) \\
& 1 / R_{\mathrm{x} 1}=1 / R_{\mathrm{cx}}+1 / R_{\mathrm{ix}} \\
& R_{\mathrm{px}}=a /\left(\mu_{0} \mu_{\mathrm{r}} b t_{\mathrm{p}}\right) \\
& R_{\mathrm{x} 2}=R_{\mathrm{px}}
\end{aligned}
$$

In the equation (3), the average magnetic path length is set to $(a+h)$ because the average magnetic path length of the side of upper shield parallel to the $\mathrm{YZ}$ plane is set to $(h / 2)$. The sides of upper shield parallel to XZ plane are not connected to those to YZ plane because the upper shield is made by bending. Therefore, the magnetic path between the sides of upper shield parallel to XZ plane and those to YZ plane is not considered in equation (3).

When the dimensions of the prototype package were substituted into these equations, the magnetic reluctance values in Equations (3) - (7) were as shown in Table 1. $R_{i x}$ was much larger than $R_{c x}$ and $R_{p X}$ so that little flux flowed in the space inside the package.

Table 1 Magnetic reluctances when $\mathrm{x}$-directional external field was applied

\begin{tabular}{cc}
\hline Part & Magnetic reluctance $\left(\mathrm{H}^{-1}\right)$ \\
\hline$R_{c x}$ & $2.0 \times 10^{5}$ \\
$R_{i x}$ & $1.0 \times 10^{9}$ \\
$R_{X 1}$ & $2.0 \times 10^{5}$ \\
$R_{p x}=R_{x 2}$ & $8.4 \times 10^{4}$ \\
\hline
\end{tabular}


Fig. 9 shows the magnetic circuit model when a $z^{-}$ directional external field was applied. The $z$-directional external field source was set to $\phi_{z} . R_{a z}$ were the magnetic reluctances of air outside the package. The gap between upper and lower shields was set to g. For magnetic flux passing through the side of the upper shield parallel to the XZ plane, the magnetic reluctance of the upper shield, that of the gap, and that of the lower shield were set to $R_{c z 1}, R_{g z 1}$, and $R_{p z 1}$, respectively. For magnetic flux passing through the side of the upper shield parallel to the YZ plane, the magnetic reluctance of the upper shield, that of the gap, and that of the lower shield were set to $R_{c z 2}, R_{g z 2}$ and $R_{p z 2}$, respectively. $R_{i z}$ was the magnetic reluctance inside the package. When the magnetic reluctance of the whole package is $R_{z}$, each magnetic reluctance can be expressed as follows.

$$
\begin{aligned}
& R_{\mathrm{cz} 1}=(\mathrm{b} / 4+h) /\left(\mu_{0} \mu_{\mathrm{r}} a t_{\mathrm{c}}\right) \\
& R_{\mathrm{gz} 1}=g /\left(\mu_{0} a t_{\mathrm{c}}\right) \\
& R_{\mathrm{pz} 1}=(b / 4) /\left(\mu_{0} \mu_{\mathrm{r}} a t_{\mathrm{p}}\right) \\
& R_{\mathrm{cz} 2}=(a / 4+h) /\left(\mu_{0} \mu_{\mathrm{r}} a t_{\mathrm{c}}\right) \\
& R_{\mathrm{gz} 2}=g /\left(\mu_{0} b t_{\mathrm{c}}\right) \\
& R_{\mathrm{pz} 2}=(a / 4) /\left(\mu_{0} \mu_{\mathrm{r}} b t_{\mathrm{p}}\right) \\
& R_{\mathrm{iz}}=(h+g) /\left(\mu_{0} a b\right) \\
& 1 /\left(R_{\mathrm{z}}\right)=2 /\left(R_{\mathrm{cz} 1}+R_{\mathrm{g} 1}+R_{\mathrm{pz} 1}\right) \\
& \quad+2 /\left(R_{\mathrm{cz} 2}+R_{\mathrm{g} 2}+R_{\mathrm{pz} 2}\right)+1 /\left(R_{i z}\right)
\end{aligned}
$$

When the dimensions of the prototype package were substituted into these equations, the magnetic reluctance values in Equations (8) - (15) were as shown in Table 2.

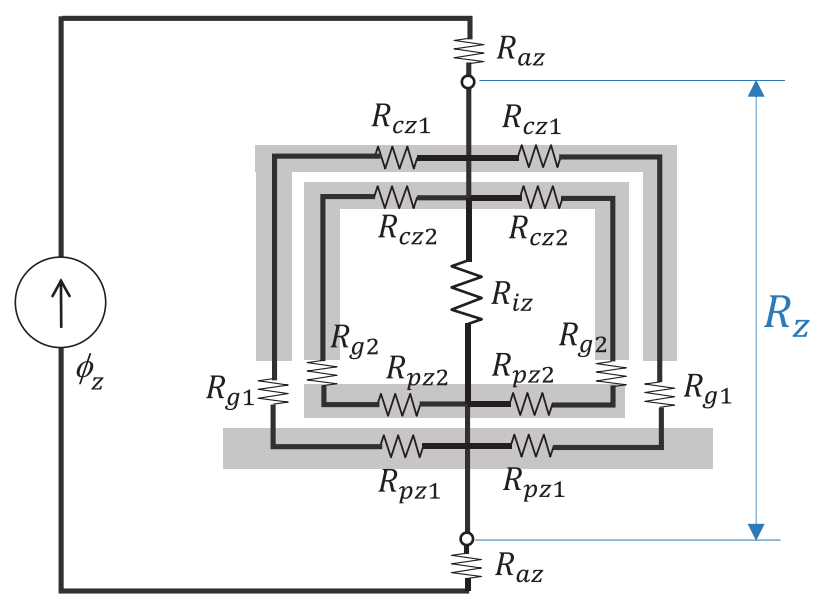

Fig. 9 Magnetic circuit model when $\mathrm{z}$-directional external field was applied
Table 2 Magnetic reluctances when z-directional external field was applied

\begin{tabular}{cc}
\hline Part & Magnetic reluctance $\left(\mathrm{H}^{-1}\right)$ \\
\hline$R_{c z 1}$ & $5.4 \times 10^{4}$ \\
$R_{g z 1}$ & $5.1 \times 10^{7}$ \\
$R_{p z 1}$ & $1.3 \times 10^{4}$ \\
$R_{c z 2}$ & $7.8 \times 10^{4}$ \\
$R_{g z 2}$ & $6.5 \times 10^{7}$ \\
$R_{p z 2}$ & $2.1 \times 10^{4}$ \\
$R_{i Z}$ & $4.7 \times 10^{7}$ \\
$R_{Z}$ & $1.1 \times 10^{7}$ \\
\hline
\end{tabular}

The magnetic reluctances in the shield, $R_{c z 1}, R_{p z 1}, R_{c z 2}$, and $R_{p z 2}$, were low. When $\mathrm{g}$ was set to 0.03 millimeters, the magnetic reluctances of gaps, $R_{g 1}$ and $R_{g 2}$, became high. The magnetic reluctance inside the package, $R_{i z}$, was the same as that of the gap between the shields. Hence, the flux did not pass only through the gap but also inside the package. This resulted in an inflow of flux intrusion as shown in Fig. 7 (b).

Additionally, $R_{i x} / R_{x 1}$ and $R_{i z} / R_{z}$ were calculated when $g$ was changed. When the area through which magnetic flux passes was not changed, $R_{i x} / R_{x 1}$ and $R_{i z} / R_{z}$ were inversely proportional to $H_{\text {in }} / H_{\text {ex }}$. This is because the magnetic field was proportional to the magnetic flux in the air. Referring to Equation (1), MSE was calculated from $H_{i n} / H_{e x}$, so $R_{i x} / R_{x 1}$ and $R_{i z} / R_{z}$ can be considered to show the same tendency as $M S E_{x x}$ and $M S E_{z z}$, respectively.

Fig. 10 shows the magnetic circuit calculation results. $R_{i x} / R_{x 1}$ did not depend on the gap, whose value was constant at $4.9 \times 10^{3}$. In comparison, $R_{i z} / R_{z}$ monotonically decreased as the gap increased.

Fig. 10 also shows the FEM analysis results for $M S E_{X X}$ and $M S E_{z z}$ when g was changed. $M S E_{x x}$ and $M S E_{z z}$ were calculated with the same model shown in Section 3.1. $M S E_{X X}$ was almost unchanged by the gap, whose value was constant at $67 \mathrm{~dB}$. In comparison, $M S E_{z z}$ was greatly influenced by the gap. When the gap was zero, $M S E_{z z}$ was as high as $62 \mathrm{~dB}$. In contrast, when the gap was more than $0.01 \mathrm{~mm}, M S E_{z z}$ is less than $17 \mathrm{~dB}$.

The magnetic circuit calculation results exhibited almost the same tendency as the FEM analysis results as shown in Fig. 10. The $R_{i x} / R_{x 1}$ of the magnetic circuit calculation was $7 \mathrm{~dB}$ higher than the $M S E_{X x}$ of the FEM analysis in the $\mathrm{X}$ direction. Because the FEM analysis calculated leakage flux and flux inflow from the hole for wires, the calculation results for the flux density inside the package of the FEM analysis were higher than for the magnetic circuit calculation. The difference of $7 \mathrm{~dB}$ at around $70 \mathrm{~dB}$ is about $1 / 4000$ of $H_{e x}$, which is a small difference.

In Fig. 10, some difference between the magnetic circuit calculation result and the FEM analysis result appear in the $\mathrm{Z}$ direction especially when the gap is small. In FEM analysis, the effective permeability of the shield is lower than that of the material due to the inclusion of magnetic flux perpendicular to the shield, which causes a decrease $M S E_{z z}$. In addition, when the gap is short and 
magnetic reluctance of gap decrease, the difference in effective permeability appear relatively large.

$M S E_{X X}$ and $M S E_{z z}$ measurement results are also shown in Fig. 10 but plotted only one point each. This is because the gap between the upper shield and the lower shield cannot be controlled in the prototype due to viscosity of the conductive paste. One the other hand, when the magnetic circuit calculation is applied to a relatively large package with the internal space of $5 \times 5 \times 2.2 \mathrm{~mm} 3$ and the gap of $0.03 \mathrm{~mm}$ in Ref. 6$), R_{i z} / R_{z}$ is $26 \mathrm{~dB}$. The measurement result of $M S E_{z z}$ showed almost the same value, $25 \mathrm{~dB}$, in Ref. 6 ), which supports the validity of the magnetic circuit model.

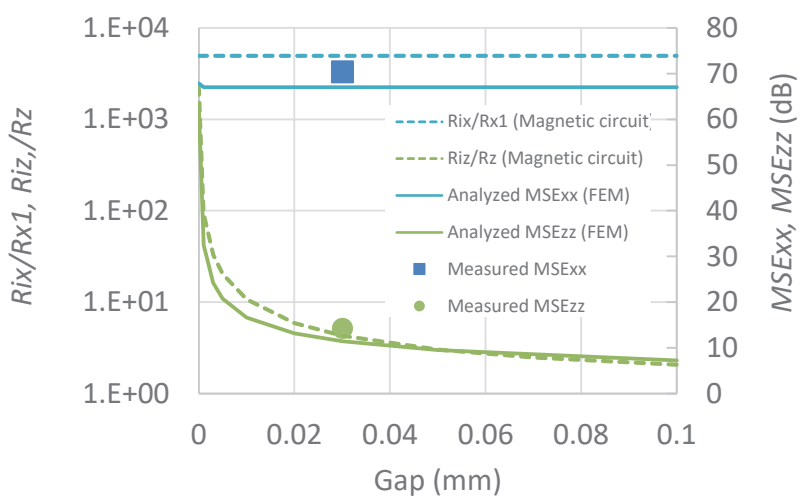

Fig. 10 Calculation results for $R_{i x} / R_{x 1}, R_{i z} / R_{z}$, FEM analysis result for MSE, and measurement results when gap between shields was changed.

\section{Conclusion}

The anisotropic MSE of a magnetic shield for small electronic components that consists of two pieces of magnetic plate was measured and analyzed. The prototype was a small package, $5.0 \times 6.0 \times 1.7 \mathrm{~mm}^{3}$, because it was assembled by using a MEMS package assembly line. The measured anisotropic MSE showed a tendency similar to that of the FEM analysis result. In addition, two magnetic circuit models were introduced to explain the cause of the anisotropic MSE. The two models differed in terms of the direction of the external field. The calculation results for the magnetic circuit models showed the same tendency as the FEM analysis results when the gap between the upper and lower shields was changed.

The two magnetic circuit models revealed the cause of the anisotropic MSE of the package. When the magnetic flux flowed in the gap between the two shields, the magnetic reluctance inside the package was almost the same as that of the gap between the shields. In this case, the magnetic flux did not flow only in the shield and the gap but also inside the package. When the magnetic flux did not flow in the gap between the two shields, the flux did not flow inside the package because the magnetic reluctance inside the package was much higher than that in the shield. The change in the flow of flux and magnetic reluctance brought about by the external field caused the anisotropic MSE.

\section{References}

1) Y.Fuji, Y. Higashi, K. Masunishi, A. Yuzawa, T. Nagata, S. Kaji, K. Okamoto, S. Baba, T. Ono, and M. Hara: J. Appl. Phys., 123, 153901 (2018)

2) T. Nakano, M. Oogane, T. Furuichi, K. Ao, H. Naganuma, and Y. Ando: IEEE Trans. Magn., 52, 4001304 (2016)

3) H. Lin, Y. Gao, X. Wang, T. Nan, M. Liu, J. Lou, G. Yang, Z. Zhou, X. Yang, J. Wu, M. Li, Z. Hu, and N. X. Sun: IEEE Trans. Magn., vol. 52, 4002208 (2016)

4) W. Wang, and Z. Jiang: IEEE Trans. Magn., 44, 4175 (2008)

5) T. Watanabe, and S. Yamamichi: Proc. IEEE 62nd Electron. Compon. Technol. Conf., 920 (2012)

6) K. Yamada: IEEE Trans. Magn., 53, 8500104 (2017)

7) E. Paperno, and I. Sasada: J. Magn. Soc. Jpn., 24, 40 (2000)

Received Sep. 12, 2019; Accepted Nov. 08, 2019 